\title{
功能多样性对典型阔叶红松林生产力的影响
}

\author{
温 纯 ${ }^{1}$ 金光泽 ${ }^{1,2 *}$ \\ ${ }^{1}$ 东北林业大学生态研究中心, 哈尔滨 $150040 ;{ }^{2}$ 东北林业大学森林生态系统可持续经营教育部重点实验室, 哈尔滨 150040
}

摘 要 为比较生物量比率假说与生态位互补假说在解释生产力变异的相对重要性, 探讨生物多样性和生产力之间的关系 是否受到生物和非生物因素的影响, 该研究依托小兴安岭 $9 \mathrm{hm}^{2}$ 阔叶红松(Pinus koraiensis)林动态监测样地, 通过计算群落初 始生物量、物种多样性、功能多样性、植物性状的群落加权平均值和测定环境因子, 运用线性回归模型、结构方程模型, 比 较了物种多样性和功能多样性与生产力的相关性。结果表明: (1)物种多样性和功能多样性均对生产力有显著作用, 功能多样 性比物种多样性与生产力的关系更为密切; (2)功能多样性指数比群落加权平均值能更好地解释生产力变异, 说明生态位互补 假说更适用于解释阔叶红松林群落内生产力的变异; (3)生物多样性与生产力的关系受生物因素与非生物因素的共同作用, 相 较于多样性和功能性状组成(植被质量), 初始林分生物量(植被数量)能更有效地解释生产力的变异。生物多样性与生产力关 系的研究应从植被质量与植被数量同时出发, 评估生态系统过程的多种非生物和生物驱动因素, 同时维护森林功能多样性, 加强植物与土壤环境的保护, 对有效增加生产力和维持生物多样性具有重要意义。

关键词＼cjkstart功能多样性; 森林生产力; 生态位互补假说; 生物量比率假说; 结构方程模型

温纯, 金光泽 (2019). 功能多样性对典型阔叶红松林生产力的影响. 植物生态学报, 43, 94-106. DOI: 10.17521/cjpe.2018.0312

\section{Effects of functional diversity on productivity in a typical mixed broadleaved-Korean pine forest}

WEN Chun ${ }^{1}$ and JIN Guang-Ze $\mathrm{e}^{1,2^{*}}$

${ }^{1}$ Center for Ecological Research, Northeast Forestry University, Harbin 150040, China; and ${ }^{2}$ Key Laboratory of Sustainable Forest Ecosystem ManagementMinistry of Education, Northeast Forestry University, Harbin 150040, China

\section{Abstract}

Aims Exploring the relationship between biodiversity and ecosystem productivity has become a hot topic in ecological research. The results support the mass ratio hypothesis and niche complementarity hypothesis, but their relative importance is still controversial. Our aim is to test the relative importance of these two hypotheses in explaining the variability of productivity, and to explore whether the relationship between biodiversity and productivity is influenced by both biotic and abiotic factors.

Methods We used the data of woody plants in a $9 \mathrm{hm}^{2}$ typical mixed broadleaved-Korean pine forest. By calculating the initial biomass, species diversity, functional diversity, community-weighted mean functional traits and measuring environmental factors, we analyzed the relationship between diversity and productivity by the linear regression model and structural equation model.

Important findings The results showed that: (1) Both species diversity and functional diversity played a significant role in productivity, and functional diversity was more closely related to productivity than species diversity; (2) the functional diversity index could better explain the variation of productivity than community-weighted mean functional traits. It suggested that the niche complementarity hypothesis was more suitable for explaining the variation of productivity in the mixed broadleaved-Korean pine forest; (3) the relationship between biodiversity and productivity was affected by biotic and abiotic factors, and compared with diversity and functional character composition (vegetation quality), initial stand biomass (vegetation quantity) could explain the variation of productivity more effectively. Our study suggests that, it is important to maintain forest functional diversity and strengthen the protection of plants and soil environments to increase productivity and biodiversity effectively.

收稿日期Received: 2018-12-13 接受日期Accepted: 2019-02-13

基金项目：国家自然科学基金(31730015)和中央高校基本科研业务费专项资金(2572017EA02)。Supported by the National Natural Science Foundation of China (31730015), and the Fundamental Research Funds for the Central Universities (2572017EA02).

* 通信作者Corresponding author (taxus@126.com) 
Key words functional diversity; forest productivity; niche complementarity hypothesis; mass ratio hypothesis; structural equation model

Wen C, Jin GZ (2019). Effects of functional diversity on productivity in a typical mixed broadleaved-Korean pine forest. Chinese Journal of Plant Ecology, 43, 94-106. DOI: 10.17521/cjpe.2018.0312

探索生物多样性与生态系统功能之间的关系已 经成为生态学领域的一个核心问题, 尤其是更好地 了解天然林地上生产力的驱动因素, 对于森林的可 持续经营十分重要(Cardinale et al., 2012; Zhang \& Chen, 2015)。众多的研究表明, 生物多样性对生态 系统功能的影响, 不仅归因于物种数量, 还依赖于 物种种类及物种所具有的功能特性(Mokany et al., 2008)。关于哪些多样性指标与地上生物量和生产力 有关，以及应如何量化，一直存在争议(Díaz et al., 2007; Ouyang et al., 2016)。以物种丰富度为代表的 物种多样性是最简单的多样性衡量标准, 通常被用 作地上生物量和生产力的生物预测指标, 但研究表 明其只能解释生产力的较小一部分变异(Zhang et al., 2012; Ruiz-Benito et al., 2014)。功能多样性是指 植物群落中功能性状的值、范围、分布或分散程度 (Laliberté \& Legendre, 2010), 主要包括功能多样性 指数与群落加权平均值 (CWM) 两部分 (黄小荣, 2018)。研究表明, 相较于物种多样性, 功能性状多 样性是解释生态系统功能变化更有效的指标, 其与 生态系统生产力的关系更为密切(Gazol \& Camarero, 2016)。

植物多样性影响生态系统功能的机理存在两种 假说。一是“生态位互补假说” (Tilman, 1997), 认为 随着多样性的增加, 群落内树种的功能特性差异增 大, 如叶面积、冠层高度、生长速度等, 能够缓解竞 争。通常用多样性指数(如物种、功能多样性)来表 征该假说。二是“生物量比率假说” (Grime, 1998), 认为某个时间点的生态系统功能主要由植物优势物 种的功能性状值决定。当该假说占主导时, 可以检 测到 CWM 是解释生态系统功能的最重要因子 (Tobner et al., 2016; Ali et al., 2017)。尽管目前研究 普遍认为生态位互补与生物量比率假说并不相互排 斥, 但二者的相对重要性还存在很大争论。Cardinale和Gonzalez (2011)整合分析多样性种植实验结 果, 发现两种假说对生产力变异的解释基本持平; Ali等(2017)则发现生物量比率假说在解释中国东部 亚热带次生林的生产力变异中占主导地位; Ratcliffe 等(2016)发现在欧洲温带森林中生态位互补和生物
量比率效应对树木生长均有促进作用，但在水分受 限制的地中海森林中生态位互补假说能够更好地解 释生产力的变异。

多样性并非生态系统生产力的唯一驱动因素, 它同时还受非生物因素和其他生物因素的影响, 如 初始生物量、土壤养分等环境因子(Lin et al., 2012; Chen et al., 2016)。有研究认为, 林分的初始地上生 物量是森林生产力的关键驱动因素, 是植被数量 (即初始生物量) 而不是植被质量(即多样性)对生产 力变异起着主要作用(Lohbeck et al., 2015)。研究发 现单纯考虑多样性与生产力二元关系时, 物种丰富 度显著影响生产力, 而整合考虑土壤肥力等非生物 因子的作用后, 二者关系变得不明显(Grace et al., 2016); 与此相反, 当去除其他非生物(海拔、冠层光 照)和生物因子(树干密度、森林演替阶段)后, 我国 亚热带森林中多样性-生产力正相关关系变弱甚至 不相关(Ouyang et al., 2016); 有研究表明, 土壤因 素推动了生产力的变异, 而生态位互补和生物量比 率机制在天然林中的重要性却微不足道(Van et al., 2017)。综上来看, 生物因素(植物多样性、初始生物 量等)与非生物因素(土壤、地形等)的相对重要性仍 需进一步验证。

阔叶红松(Pinus koraiensis)林是我国东北东部 山区的地带性顶极植被, 了解阔叶红松林植物多样 性与生产力的关系, 可为次生林的顶极植被恢复提 供科学依据。本研究以 $9 \mathrm{hm}^{2}$ 典型阔叶红松林动态监 测样地为研究对象, 利用线性模型和结构方程模型 (SEM), 基于群落地上生产力、地上初始生物量、 4 个物种多样性指标、 11 个功能多样性指标、 8 种植物 性状的群落加权平均值以及地形与土壤环境因子数 据, 试图回答以下3个问题: (1)功能多样性是否比物 种多样性能更好地解释植物群落地上生产力的变异? (2)群落加权平均值(生物量比率假说)与多样性指数 (生态位互补假说) 哪一个能更好地解释阔叶红松林 生产力的变异? (3)多样性与生产力的关系是否受其 他生物和非生物因素的共同作用? 两者的相对重要 性如何? 


\section{1 材料和方法}

\section{1 研究样地概况}

调查样地设在黑龙江凉水国家级自然保护区 $\left(47.18^{\circ} \mathrm{N}, 128.89^{\circ} \mathrm{E}\right)$ 。该保护区位于黑龙江省伊春 市带岭区, 地处小兴安岭南坡达里带岭支脉东坡, 地形较为复杂, 海拔280-707 m, 为典型的低山丘 陵地貌。该区属于温带大陆性季风气候, 年平均气 温 $-0.3{ }^{\circ} \mathrm{C}$, 年平均最高气温 $7.5{ }^{\circ} \mathrm{C}$, 年平均最低气 温-6.6 ${ }^{\circ} \mathrm{C}$ 。地带性土壤为暗棕壤, 非地带性土壤为 草甸土、沼泽土和泥炭土。本地区的阔叶红松林以 红松为建群种, 伴生的温性阔叶树种有水曲柳(Fraxinus mandschurica)、白桦(Betula platyphylla)、紫椴 (Tilia amurensis)、色木槭(Acer mono)、花楷槭(Acer ukurunduense)等, 寒温性树种以臭冷杉(Abies nephrolepis)、红皮云杉(Picea koraiensis)为主, 灌木有毛 榛(Corylus mandshurica)、东北山梅花(Philadelphus schrenkii)和刺五加(Acanthopanax senticosus)等。

\section{2 数据调查与生产力计算}

以 $10 \mathrm{~m} \times 10 \mathrm{~m}$ 连续样方为取样单元, 将 $9 \mathrm{hm}^{2}$ 阔叶红松林动态监测样地划分为 900 个样方。于 2005 年对样地中所有胸径 $\geqslant 2 \mathrm{~cm}$ 的木本植物的物种、胸 径、树高、冠幅、相对位置和状态(存活、倒伏、枯 立)进行记录并挂牌监测。样地每隔 5 年进行复查, 2010年完成第一次复查, 2015年完成第二次复查。 2015年复查时样地内胸径 $\geqslant 2 \mathrm{~cm}$ 的活立木共 17161 株, 隶属于 20 科 34 属 48 种。

根据东北主要林木生物量的异速生长方程 (陈传国和朱俊风, 1989), 用实测胸径计算木本植 株的地上生物量。群落木本植物生产力 (CWP, $\left.\mathrm{Mg} \cdot \mathrm{hm}^{-2} \cdot \mathrm{a}^{-1}\right)$ 的计算参考了 Chave等(2003)的方法: $C W P=(G+R) / 0.01 \times T$, 式中 $G$ 为 $10 \mathrm{~m} \times 10 \mathrm{~m}$ 样方 内两次调查都存活个体地上生物量的增长量; $R$ 为 $10 \mathrm{~m} \times 10 \mathrm{~m}$ 样方内第一次调查胸径小于 $2 \mathrm{~cm}$, 复查 时胸径超过 $2 \mathrm{~cm}$ 个体的地上生物量总和, $T$ 为两次 调查时间的间隔(10年)。

\section{3 叶片功能性状与环境变量测定}

本研究测定与植物生长和群落生产力密切相关 的8种功能性状: 木材密度、最大树高、比叶面积、 叶面积、叶片厚度、叶片干物质含量、叶片碳含量 和叶片氮含量(Finegan et al., 2015; Van et al., 2017) (表1)。对 41 种木本植物的功能性状进行了测定, 这些物种的生物量总和占群落总生物量的 $85 \%$ 以上
(吕亭亭等, 2014)。每个物种随机选取 5 株生长良好、 无病虫害的植株，每株剪下 20 片健康无损、被日光 照射无遮阴的叶片, 依据植物性状标准化测定方法 (Cornelissen et al., 2003), 测定6种叶片性状; 在样 地周围对每个物种随机选择 3 株健康植株, 在距离 地面 $1.3 \mathrm{~m}$ 处钻取一个生长芯, 把生长芯分成 $1 \mathrm{~cm}$ 的 片段，用水置换法测定各节段鲜体积，103 ${ }^{\circ} \mathrm{C}$ 烘干 $72 \mathrm{~h}$, 用烘干的质量除以鲜体积来测量木材密度; 最大树高来源于《中国植物志》(Wu \& Raven, 19942009)。

地形变量包括凹凸度、海拔和坡度, 将 $9 \mathrm{hm}^{2}$ 阔 叶红松林动态监测样地划分为 225 个 $20 \mathrm{~m} \times 20 \mathrm{~m}$ 小 样方, 样方所有变量均根据每个小样方单元的四个 角的高度计算(Chang et al., 2013)。在每个 $20 \mathrm{~m} \times 20 \mathrm{~m}$ 样方原点位置, 取 1 个样点, 同时随机选择一个方向, 在离原点 $2 、 5 、 8 \mathrm{~m}$ 处选任意 2 个样点, 取土壤表层 $(0-10 \mathrm{~cm})$ 的样品, 于实验室内测定速效氮、速效磷、 速效钾、全氮、全磷、有机碳含量、 $\mathrm{pH}$ 值、土壤容 重、体积含水率和质量含水率。我们将每个 $20 \mathrm{~m} \times$ $20 \mathrm{~m}$ 的样方分为了 16 个 $5 \mathrm{~m} \times 5 \mathrm{~m}$ 的子样方后，应用 ArcGIS 10.1通过地统计学分析工具中的 ordinary Kriging方法对地形和土壤因子共13项指标进行空 间插值，从而获得每个 $10 \mathrm{~m} \times 10 \mathrm{~m}$ 取样单元的生境 因子指标(表2)。

\section{4 多样性指数与群落加权平均值计算}

计算每个样方的物种多样性，包括物种丰富度 $(C)$ 、辛普森指数 $(D)$ 、香农指数 $\left(H^{\prime}\right)$ 、均匀度指数 $(J)$, 各指数计算公式(孙儒泳等, 2002)如下:

$$
\begin{aligned}
& C=S \\
& D=1-\sum_{i=1}^{S} P_{i}^{2} \\
& P_{i}^{2}=\frac{n_{i}\left(n_{i}-1\right)}{N(N-1)} \\
& H^{\prime}=-\sum_{i=1}^{S} P_{i} \ln P_{i} \\
& J=H / \ln S
\end{aligned}
$$

式中, $S$ 为群落中物种数; $N$ 为所有物种的个体数之 和; $P_{i}$ 为种 $i$ 的个体在全部个体中的比例; $n_{i}$ 为第 $i$ 个 物种的个体数。

基于所测定的 8 个植物功能性状，计算每个小 样方的功能均匀度 $(F E v e) 、 R a O$ 二次熵指数 $\left(F D_{Q}\right)$ 、 功能分散指数(FDis), 计算基于 8 个功能性状组合的 
表1 与植物生长和群落生产力相关的 8 种功能性状及其意义

Table 1 Eight functional traits related to plant growth and community woody productivity and their significance

\begin{tabular}{ll}
\hline 功能性状 Functional trait & 功能意义 Functional significance \\
\hline 木材密度 Wood density $(W D)\left(\mathrm{g} \cdot \mathrm{mm}^{-3}\right)$ & 木材经济谱, 生长和生存之间的权衡, 水的运输和分配 \\
Wood economic spectrum, trade-off between growth, transport and distribution of water \\
最大高度 Maximum height $(M H)(\mathrm{m})$ & 植物竞争活力与策略, 光生态位, 结构多样性 \\
& Plant competitive vigor and strategy, light niche, structural diversity \\
比叶面积 Specific leaf area $(S L A)\left(\mathrm{mm}^{2} \cdot \mathrm{g}^{-1}\right)$ & 叶经济谱, 植物耐荫性 Leaf economic spectrum, plant shade tolerance \\
叶面积 Leaf area $(L A)\left(\mathrm{mm}^{2}\right)$ & 光竞争, 蒸腾速率 Light acquisition, transpiration rate \\
叶片厚度 Leaf thickness $(L T)(\mathrm{mm})$ & 储水能力, 蒸腾速率 Storage capacity, transpiration rate \\
叶片干物质含量 & 养分吸收, 结构物质 Nutrient absorption, structural substance \\
Leaf dry matter content $(L D M C)\left(\mathrm{mg} \cdot \mathrm{g}^{-1}\right)$ & 结构物质, 养分吸收 Structural substance, nutrient absorption \\
叶片碳含量 & 叶经济谱, 氮吸收 Leaf economic spectrum, nitrogen acquisition \\
Leaf carbon concentration $(L C C)\left(\mathrm{mg} \cdot \mathrm{g}^{-1}\right)$ & \\
叶片氮含量 & \\
Leaf nitrogen concentration $(L N C)\left(\mathrm{mg} \cdot \mathrm{g}^{-1}\right)$ & \\
\hline
\end{tabular}

表2 阔叶红松林 $9 \mathrm{hm}^{2}$ 样地内基础环境变量信息统计表

Table 2 Summary of environment variables in a $9 \mathrm{hm}^{2}$ mixed broadleaved-Korean pine forest plot

\begin{tabular}{|c|c|c|c|}
\hline 变量 Variables & 范围 Range & 平均值 Mean & 标准偏差 $S D$ \\
\hline 凹凸度 Convexity & $-1.91-2.36$ & 0.01 & 0.40 \\
\hline 海拔 Elevation (m) & $425.45-505.52$ & 463.25 & 18.20 \\
\hline 坡度 Slope $\left(^{\circ}\right)$ & $3.30-38.46$ & 15.91 & 6.49 \\
\hline 速效氮含量 Soil available nitrogen content $\left(\mathrm{mg} \cdot \mathrm{kg}^{-1}\right)$ & $605.77-1482.85$ & 1103.60 & 161.34 \\
\hline 速效磷含量 Soil available phosphorus content $\left(\mathrm{mg} \cdot \mathrm{kg}^{-1}\right)$ & $2.54-61.96$ & 8.83 & 6.96 \\
\hline 速效钾含量 Soil available potassium content $\left(\mathrm{mg} \cdot \mathrm{kg}^{-1}\right)$ & $190.46-516.66$ & 332.61 & 59.05 \\
\hline 全氮含量 Soil total nitrogen content $\left(\mathrm{g} \cdot \mathrm{kg}^{-1}\right)$ & $4.60-12.28$ & 8.40 & 1.60 \\
\hline 全磷含量 Soil total phosphorus content $\left(\mathrm{g} \cdot \mathrm{kg}^{-1}\right)$ & $0.40-1.19$ & 0.82 & 0.15 \\
\hline $\mathrm{pH}$ 值 Soil pH & $5.34-6.29$ & 5.77 & 0.16 \\
\hline 有机碳含量 Soil organic carbon content (\%) & $31.29-216.77$ & 75.75 & 31.25 \\
\hline 土壤容重 Soil bulk density $\left(\mathrm{mg} \cdot \mathrm{m}^{-3}\right)$ & $0.41-0.87$ & 0.62 & 0.10 \\
\hline 体积含水率 Soil bulk moisture content (\%) & $13.72-48.40$ & 30.35 & 5.91 \\
\hline 质量含水率 Soil mass moisture content (\%) & $0.54-1.51$ & 0.91 & 0.23 \\
\hline
\end{tabular}

功能分散指数 $\left(F D_{\text {com }}\right)$ 和分别基于单个功能性状的 功能分散指数 $\left(F D_{\mathrm{WD}}, F D_{\mathrm{MH}}, F D_{\mathrm{SLA}}, F D_{\mathrm{LA}}, F D_{\mathrm{LT}}\right.$, $F D_{\mathrm{LDMC}}, F D_{\mathrm{LCC}}$ ), 同时计算了 8 个性状的群落加权平 均值(CWM)。各指数计算公式(黄小荣, 2018)如下:

$$
\begin{aligned}
& E W_{b}=\frac{d_{i j}}{w_{i}+w_{j}} \\
& P E W_{b}=\frac{E W_{b}}{\sum_{b-1}^{S-1} E W_{b}} \\
& P W_{v e}=\frac{\sum_{b-1}^{S-1} \min \left(P E W_{b}, \frac{1}{S-1}\right)-\frac{1}{S-1}}{1-\frac{1}{S-1}} \\
& F D_{Q}=\sum_{i=1}^{S} \sum_{i=1}^{S} d_{i j} w_{i} w_{j}
\end{aligned}
$$

$$
\begin{aligned}
F D_{i s} & =\frac{\sum_{j} w_{j}}{\sum_{S} w_{j}} \\
C W M & =\sum_{i=1}^{S} w_{i} \times \text { trait }_{i}
\end{aligned}
$$

式中, $S$ 为物种数目, $P E W_{b}$ 为偏加权均匀度; $E W_{b}$ 为 加权均匀度; $w_{i}$ 和 $w_{j}$ 分别为物种 $i$ 和物种 $j$ 的相对多度, $d_{i j}$ 为物种 $i$ 与物种 $j$ 间的欧氏距离; $z_{j}$ 为物种 $j$ 到加权质 心的距离; trait $_{i}$ 为物种 $i$ 的性状值。

采用R 3.5.1 vegan包的diversity函数(Oksanen et al., 2009), 计算每个样方的物种多样性指标; FD包 计算功能多样性指数与群落加权平均值(Laliberté \& Legendre, 2010)(表3)。

\section{5 数据分析}

回归分析前，为了使响应变量呈正态分布，对 
表3 阔叶红松林 $9 \mathrm{hm}^{2}$ 功能多样性指数、物种多样性指数及群落加权平均值(CWM)信息统计表

Table 3 Summary of functional diversity, species diversity indexes and community-weighted mean (CWM) functional traits in a $9 \mathrm{hm}^{2}$ mixed broadleavedKorean pine forest plot

\begin{tabular}{|c|c|c|c|}
\hline 假说 Hypothesis & 指数 Index & 平均值 Mean & 范围 Range \\
\hline \multirow{8}{*}{$\begin{array}{l}\text { 生物量比率假说 } \\
\text { Mass ratio hypothesis }\end{array}$} & 木材密度的群落加权平均值 $C W M$ of wood density $\left(C W M_{\mathrm{WD}}\right)\left(\mathrm{g} \cdot \mathrm{mm}^{-3}\right)$ & 0.47 & $0.34-0.63$ \\
\hline & 最大树高的群落加权平均值 $C W M$ of maximum height $\left(C W M_{\mathrm{MH}}\right)(\mathrm{m})$ & 17.24 & $4.59-43.01$ \\
\hline & 比叶面积的群落加权平均值 $C W M$ of specific leaf area $\left(C W M_{\mathrm{SLA}}\right)\left(\mathrm{mm}^{2} \cdot \mathrm{g}^{-1}\right)$ & 254.30 & $158.29-392.23$ \\
\hline & 叶面积的群落加权平均值 $C W M$ of leaf area $\left(C W M_{\mathrm{LA}}\right)\left(\mathrm{mm}^{2}\right)$ & 33.87 & $8.20-74.54$ \\
\hline & 叶片厚度的群落加权平均值 $C W M$ of leaf thickness $\left(C W M_{\mathrm{LT}}\right)(\mathrm{mm})$ & 0.18 & $0.08-0.44$ \\
\hline & 叶干物质含量的群落加权平均值 $C W M$ of leaf dry matter content $\left(C W M_{\mathrm{LDMC}}\right)\left(\mathrm{mg} \cdot \mathrm{g}^{-1}\right)$ & 0.29 & $0.19-0.38$ \\
\hline & 叶片碳含量的群落加权平均值 $C W M$ of leaf carbon content $\left(C W M_{\mathrm{LCC}}\right)\left(\mathrm{mg} \cdot \mathrm{g}^{-1}\right)$ & 499.22 & $446.90-563.74$ \\
\hline & 叶片氮含量的群落加权平均值 $C W M$ of leaf nitrogen content $\left(C W M_{\mathrm{LNC}}\right)\left(\mathrm{mg} \cdot \mathrm{g}^{-1}\right)$ & 23.56 & $17.49-32.38$ \\
\hline \multirow{15}{*}{$\begin{array}{l}\text { 生态位互补假说 } \\
\text { Niche complementarity } \\
\text { hypothesis }\end{array}$} & 物种丰富度 Species richness $(C)$ & 6.42 & $2-13$ \\
\hline & 香农指数 Shannon index $\left(H^{\prime}\right)$ & 1.45 & $0.41-2.24$ \\
\hline & 辛普森指数 Simpson index $(D)$ & 0.71 & $0.24-0.88$ \\
\hline & 均匀度指数 Evenness index $(J)$ & 0.89 & $0.42-1.00$ \\
\hline & 多维功能均匀度指数 Multidimensional functional evenness index (FEve) & 0.72 & $0.21-0.99$ \\
\hline & Rao二次熵指数 Rao's quadratic entropy index $\left(F D_{Q}\right)$ & 8.39 & $0.81-15.41$ \\
\hline & 8 个性状组合的功能分散指数 FD is based on eight traits combined ( $\left.F D_{\text {com }}\right)$ & 2.68 & $0.72-3.87$ \\
\hline & 木材密度的功能分散指数 $\mathrm{FD}$ is based on wood density $\left(F D_{\mathrm{WD}}\right)$ & 0.48 & $0.01-1.13$ \\
\hline & 最大高度的功能分散指数 FD is based on maximum height $\left(F D_{\mathrm{MH}}\right)$ & 0.78 & $0.07-1.71$ \\
\hline & 比叶面积的功能分散指数 FD is based on specific leaf area $\left(F D_{\mathrm{SLA}}\right)$ & 0.63 & $0.02-1.61$ \\
\hline & 叶面积的功能分散指数 $\mathrm{FD}$ is based on leaf area $\left(F D_{\mathrm{LA}}\right)$ & 0.66 & $0.02-1.61$ \\
\hline & 叶片厚度的功能分散指数 $\mathrm{FD}$ is based on leaf thickness $\left(F D_{\mathrm{LT}}\right)$ & 0.58 & $0.03-1.51$ \\
\hline & 叶干物质含量的功能分散指数 FD is based on leaf dry matter content ( $F D_{\text {LDMC }}$ ) & 0.58 & $0.06-1.26$ \\
\hline & 叶片碳含量的功能分散指数 $\mathrm{FD}$ is based on leaf carbon content $\left(F D_{\mathrm{LCC}}\right)$ & 0.93 & $0.03-2.37$ \\
\hline & 叶片氮含量的功能分散指数 $\mathrm{FD}$ is based on leaf nitrogen content $\left(F D_{\mathrm{LNC}}\right)$ & 0.51 & $0.03-2.38$ \\
\hline
\end{tabular}

各个性状的群落加权平均值单位与表1所对应的功能性状单位相同。

Community weight mean for each trait is the same as the unit of functional trait corresponding to Table 1.

其进行对数转换。用Z-score方法对解释变量进行标 准化处理, 使所有变量的范围在类似的尺度上具有 可比性。利用转换后的响应变量和解释变量构建线 性回归模型。采用R 3.5.1进行数据处理和统计分析。

\subsection{1 多重共线性检验}

多重共线性会影响解释变量对响应变量的解释 和预测能力。利用方差膨胀因子(VIF)检验变量间的 多重共线性，根据以往经验，VIF $<10$ 时认为变量间 没有多重共线性(Fox \& Monette, 1992; Fox, 2008)。 验证结果显示, 解释变量均匀度指数 $(J)$ 、叶片厚度 的群落加权平均值 $\left(C W M_{\mathrm{LT}}\right)$ 和叶片厚度的功能分散 指数 $\left(F D_{\mathrm{LT}}\right)$ 存在多重共线性, 故删去上述变量。

\subsection{2 回归线性模型构建}

将生产力作为响应变量, 物种多样性指数、功 能多样性指数、物种多样性指数及功能多样性指数、 群落加权平均值、功能多样性指数及群落加权平均 值、环境变量作为解释变量, 分别与生产力构建线
性回归模型, 并比较各模型中解释变量与生产力的 相关性。

对于包含不同指数的 6 个模型, 利用决定系数 $\left(R^{2}\right)$ 和赤池信息量准则 (AIC) 判断模型优度, 比较 物种多样性、功能多样性与生产力的相关性, 判断 物种多样性与功能多样性对森林生产力影响的差 异。

\subsection{3 变量篮选}

采用MuMIn包中的 dredge 函数(Bartoń, 2018), 分别将生产力与多样性指数的模型、生产力与群落 加权平均值的模型、生产力与环境变量的模型的各 个变量之间所有可能的组合进行拟合，计算出每个 回归模型的变量组合中各单一子模型在模型中对响 应变量解释的重要性。在拟合的模型中篎选出AIC 差值小于 2 的所有模型, 选择变量重要性最高、与生 产力最为相关的多样性指数与环境因子, 用来构建 结构方程模型。 


\subsection{4 结构方程模型构建}

利用结构方程模型分析多样性指数、环境因子 与森林生产力的直接和间接关系, 并比较生物量比 率假说与生态位互补假说在解释阔叶红松林生产力 变异的相对重要性。在构建模型之前, 需要一个先 验假设。在多样性和生产力之间已知理论的因果关 系的基础上构建初始模型, 同时考虑初始林分地上 生物量的影响和环境因素的影响。假设: (1)地形和 土壤条件在决定生物因子中起着基础性作用; (2)地 形对土壤产生影响; (3)多样性和群落加权平均值直 接影响初始林分生物量和生产力; (4)初始林分生物 量直接影响森林生产力。

根据变量筛选结果, 选择变量重要性最高、与 生产力最为相关的多样性指数与潜在环境变量, 纳 入到结构方程模型中。采用最大似然法对结构方程 模型进行拟合, 利用比较拟合指数 $(C F I)$ 、标准均方 根残差 $(S R M R) 、$ 渐进残差均方和平方根 $(R M S E A) 、$ 显著性概率值 $(p)$ 进行模型优度的评价 (Howard, 2013 )。拟合优度的临界值为 $C F I>0.9, S R M R<0.05$, $R M S E A<0.08, p>0.05$ 。利用模型中各路径标准化 系数, 定量表示不同因素对生产力的相对作用大小, 量化各个变量对森林地上生产力的直接、间接和总 标准化效应(Edwards \& Lambert, 2007), 从而比较 生态位互补假说与生物量比率假说对阔叶红松林的 适用性。上述分析利用lavaan包完成(Rosseel, 2012)。

\section{2 结果和分析}

\section{1 生产力与多样性、环境因子的相关性}

回归分析表明, 物种丰富度、香农指数、辛普 森指数与生产力极显著正相关 $(p<0.001)$ (图1); 功 能多样性指标(包括 10 个功能多样性指数和 7 个群落
加权平均值)中功能均匀度指数与生产力无显著的 相关关系 $(p=0.163)$, 其余功能多样性指数和群落 加权平均值与生产力显著相关(图2, 图3); 土壤因 子中有机碳含量、全磷含量、体积含水率与生产力 显著相关 $(p<0.05)$, 地形因子中凹凸度、海拔与生 产力显著相关 $(p<0.05)$ (图4)。

\section{2 多样性-生产力模型评价}

多样性与生产力的线性回归模型中, 功能多样 性指数与群落地上生产力的多元回归模型(模型 $\mathrm{A}$ )、 群落加权平均值与群落地上生产力的多元回归模型 (模型 $\mathrm{B}) 、$ 功能多样性指数及群落加权平均值与群落 地上生产力的多元回归模型 (模型 $\mathrm{C}$ )的决定系数 $\left(R^{2}=0.269, R^{2}=0.188, R^{2}=0.367\right)$ 均高于物种多样 性指数与群落地上生产力的多元回归模型的决定系 数 $\left(R^{2}=0.169\right)$, 而模型 $\mathrm{A} 、 \mathrm{~B} 、 \mathrm{C}$ 的 $\mathrm{AIC}$ 值 $(\mathrm{AIC}=$ 2 047.73, $\mathrm{AIC}=2$ 136.52, $\mathrm{AIC}=1$ 932.43)均低于物 种多样性指数模型的AIC值 $(\mathrm{AIC}=2$ 148.61)(表4)。

\section{3 线性模型篮选解释生产力的最佳变量}

当变量较多时，结构方程模型不易收玫，故逐 个加入多样性指标及环境变量构建结构方程模型, 构建出最优模型。经过变量篮选比较(表5), 最终以 $F D_{Q}$ 代表多样性, 叶片碳含量的群落加权平均值 $\left(C W M_{\mathrm{LCC}}\right)$ 代表群落加权平均值, 海拔代表地形变 量, 土壤全磷含量代表土壤变量, 构建最优结构方 程模型(图5), 最优结构方程模型的 $C F I=1.000$, $S R M R=0.011, R M S E A=0.017, p$ 值为 0.260 。

\section{4 各因素对群落生产力的影响}

各变量解释地上生物量变化的 $17.6 \%$, 解释群 落生产力变异的 $37.2 \%$ (图5)。功能多样性指数对生 产力有极显著的直接影响 $(p<0.001)$, 反映作用大 小的路径系数为 0.302 ; 群落加权平均值对生产力
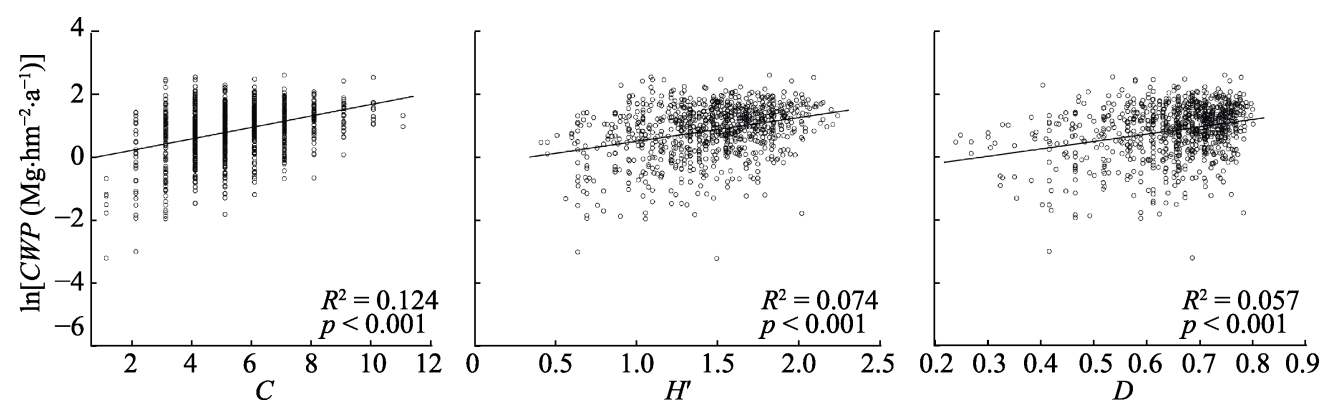

图1 典型阔叶红松林群落地上生产力 ( $\ln$ 转换后)与物种多样性指数的关系。CWP、C、H、D分别表示群落地上生产力、物 种丰富度、香农指数和辛普森指数。

Fig. 1 Relationships between coarse woody productivity (ln transformed) and species diversity index of communities in a typical mixed broadleaved-Korean pine forest. $C W P, C, H^{\prime}, D$ represent coarse woody productivity, species richness, Shannon index and Simpson index, respectively. 

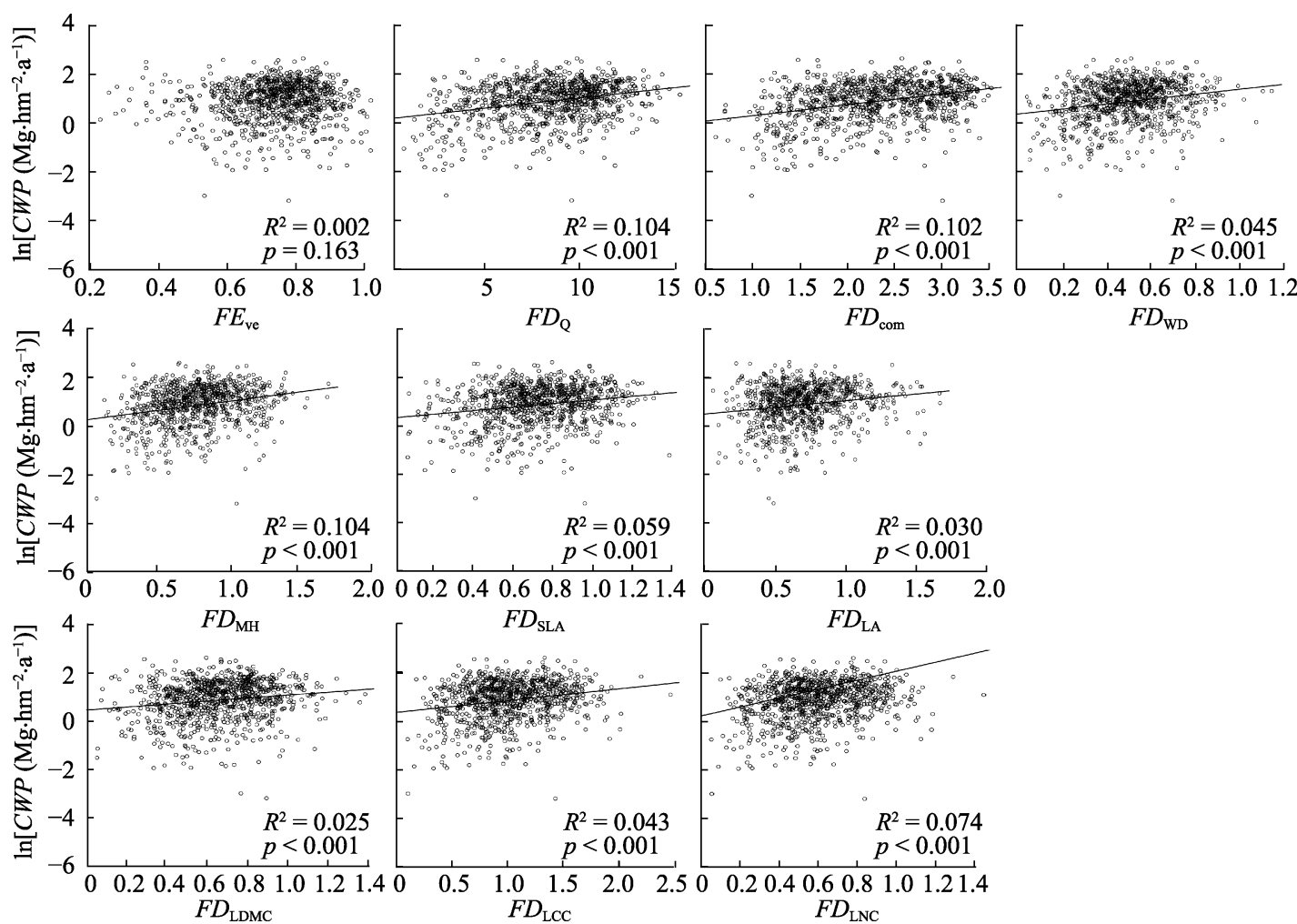

图2 典型阔叶红松林群落地上生产力 ( $\ln$ 转换后)与功能多样性指数的关系。CWP, 群落地上生产力; FEve, 多维功能均匀度指 数; $F D_{\mathrm{Q}}$, Rao二次熵指数; $F D_{\mathrm{com}}, 8$ 个性状组合的功能分散指数; $F D_{\mathrm{WD}}$, 木材密度的功能分散指数; $F D_{\mathrm{MH}}$, 最大高度的功能分 散指数; $F D_{\mathrm{SLA}}$, 比叶面积的功能分散指数; $F D_{\mathrm{LA}}$, 叶面积的功能分散指数; $F D_{\mathrm{LDMC}}$, 叶干物质含量的功能分散指数; $F D_{\mathrm{LCC}}$, 叶片碳含量的功能分散指数; $F D_{\mathrm{LNC}}$, 叶片氮含量的功能分散指数。

Fig. 2 Relationships between coarse woody productivity (ln transformed) and functional diversity index of communities in a typical mixed broadleaved-Korean pine forest. CWP, coarse woody productivity; FEve, multidimensional functional evenness index; $F D_{Q}$, Rao's quadratic entropy index; $F D_{\text {com }}$, functional dispersion indices of eight trait combinations; $F D_{\mathrm{WD}}$, functional dispersion indices of wood density; $F D_{\mathrm{MH}}$, functional dispersion indices of maximum height; $F D_{\mathrm{SLA}}$, functional dispersion indices of specific leaf area; $F D_{\mathrm{LA}}$, functional dispersion indices of leaf area; $F D_{\mathrm{LDMC}}$, functional dispersion indices of leaf dry matter content; $F D_{\mathrm{LCC}}$, functional dispersion indices of leaf carbon content; $F D_{\mathrm{LNC}}$, functional dispersion indices of leaf nitrogen content.
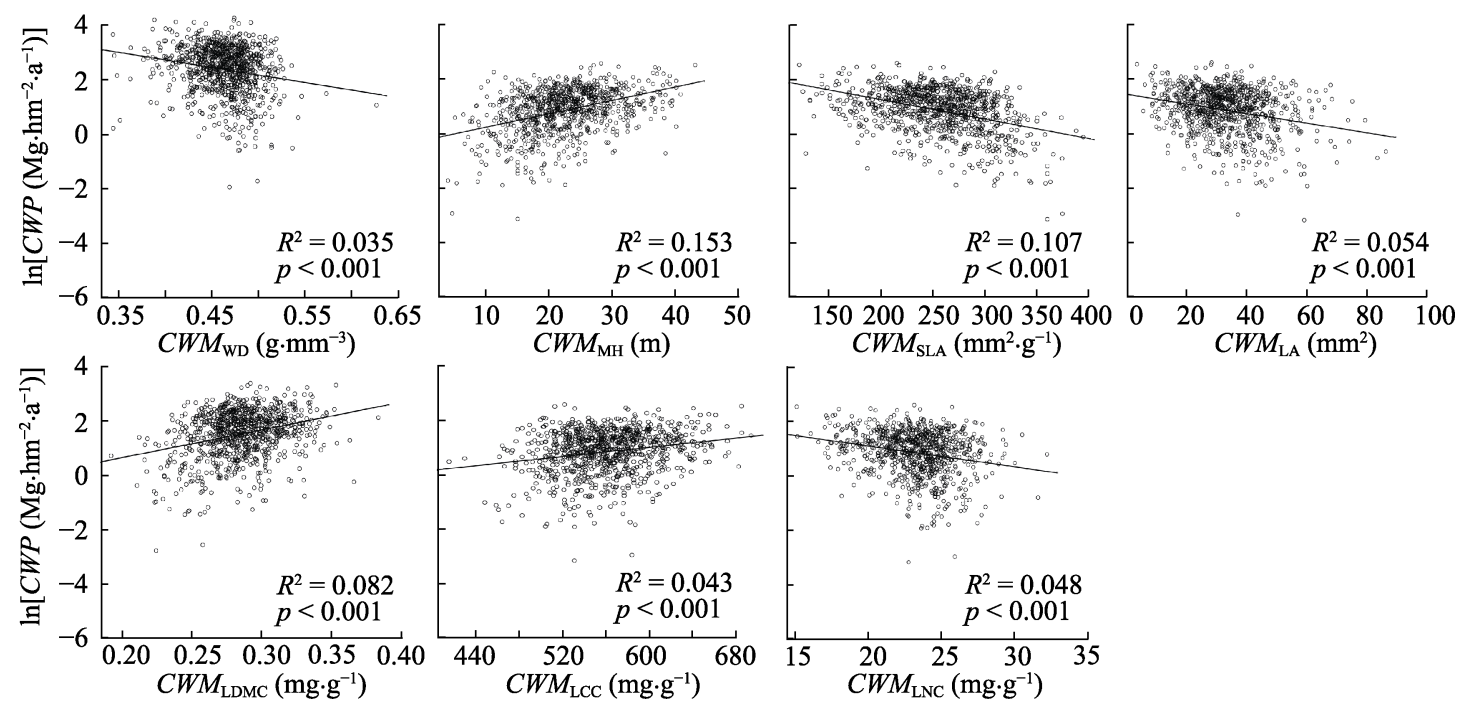

图3 典型阔叶红松林群落地上生产力 $\left(\ln\right.$ 转换后)与群落加权平均值的关系。CWP, 群落地上生产力; $C W M_{\mathrm{WD}}$, 木材密度的群落 加权平均值; $C W M_{\mathrm{MH}}$, 最大树高的群落加权平均值; $C W M_{\mathrm{SLA}}$, 比叶面积的群落加权平均值; $C W M_{\mathrm{LA}}$, 叶面积的群落加权平均值; $C W M_{\mathrm{LDMC}}$, 叶干物质含量的群落加权平均值; $C W M_{\mathrm{LCC}}$, 叶片碳含量的群落加权平均值; $C W M_{\mathrm{LNC}}$, 叶片氮含量的群落加权平均值。

Fig. 3 Relationships between coarse woody productivity (ln transformed) and community weighted mean of different traits of communities in a typical mixed broadleaved-Korean pine forest. $C W P$, coarse woody productivity; $C W M_{\mathrm{WD}}$, $C W M$ of wood density; $C W M_{\mathrm{MH}}, C W M$ of maximum height; $C W M_{\mathrm{SLA}}, C W M$ of specific leaf area; $C W M_{\mathrm{LA}}, C W M$ of leaf area; $C W M_{\mathrm{LDMC}}, C W M$ of leaf dry matter content; $C W M_{\mathrm{LCC}}, C W M$ of leaf carbon content; $C W M_{\mathrm{LNC}}, C W M$ of leaf nitrogen content.

www.plant-ecology.com 

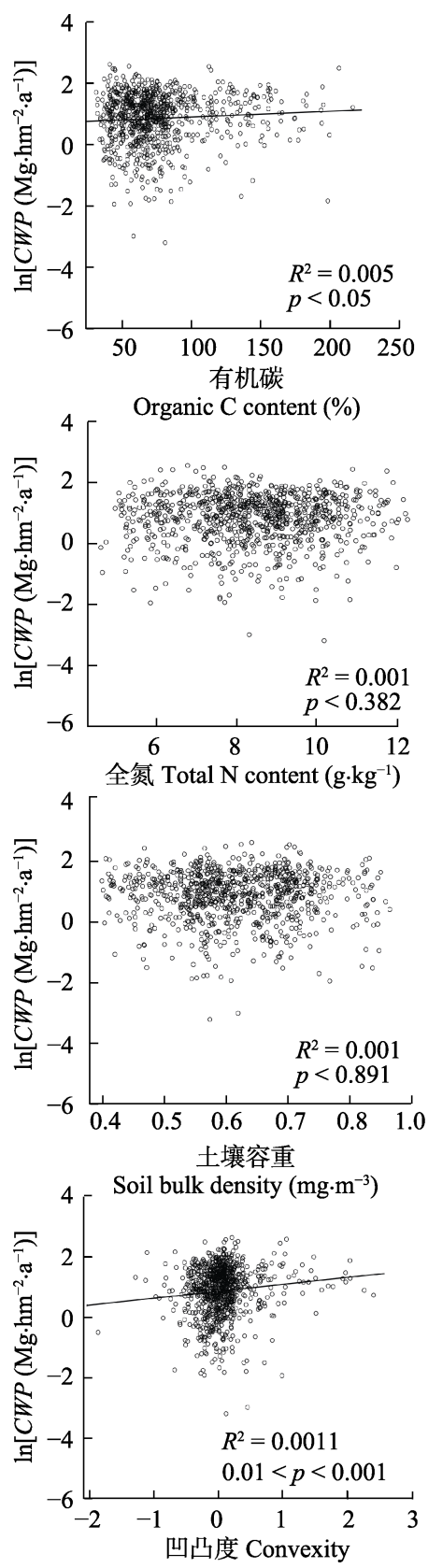
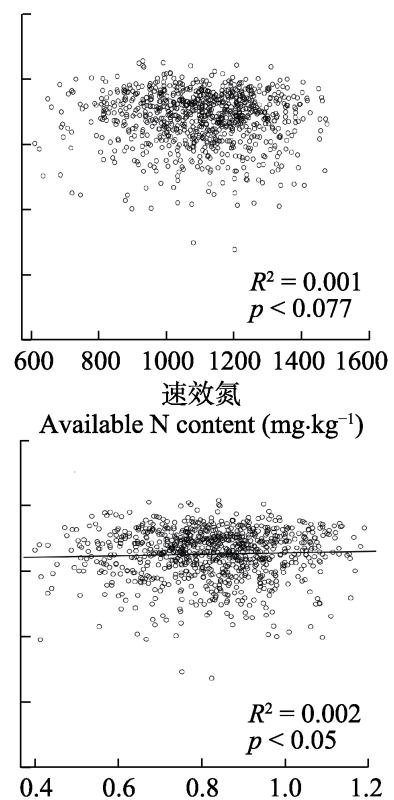

全磷 Total P content $\left(\mathrm{g} \cdot \mathrm{kg}^{-1}\right)$

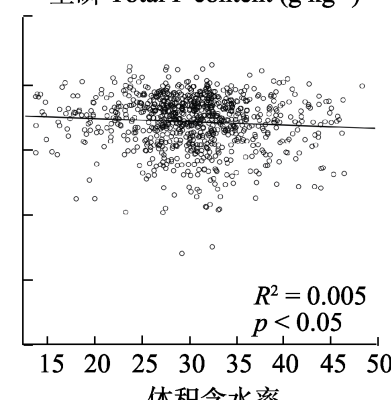

体积含水率

Bulk moisture content (\%)

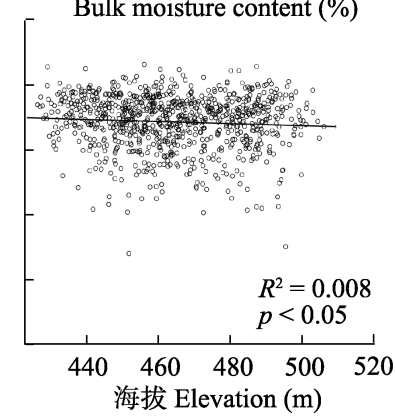

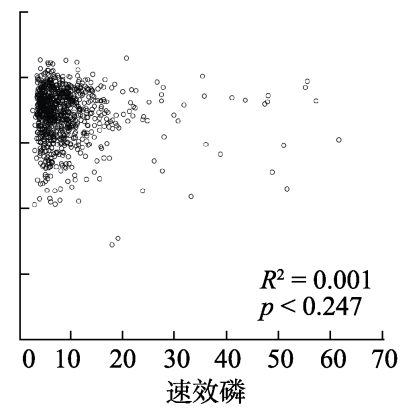
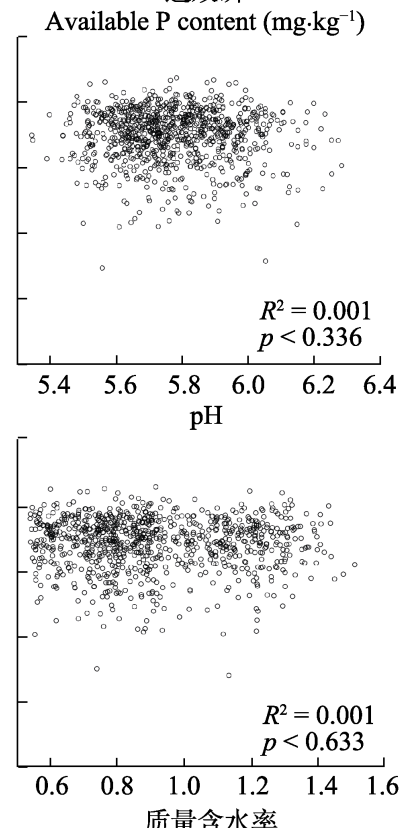

Mass moisture content (\%)

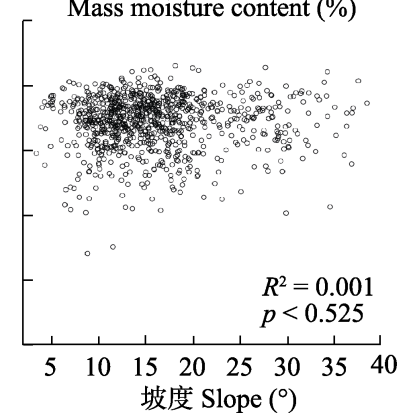

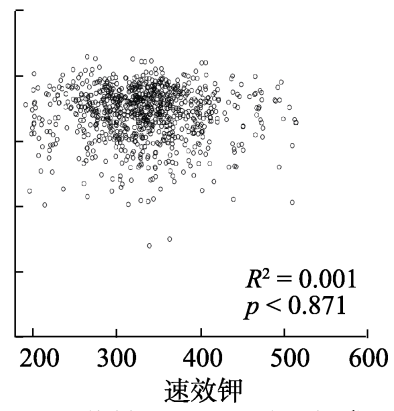

Available K content $\left(\mathrm{mg} \cdot \mathrm{kg}^{-1}\right)$

图4 典型阔叶红松林群落地上生产力 $(\ln$ 转换后, $C W P)$ 与各环境因子的关系。

Fig. 4 Relationships between coarse woody productivity (ln transformed, CWP) and environmental factors in a typical mixed broadleaved-Korean pine forest.

表4＼cjkstart典型阔叶红松林群落生产力与多样性构建的线性回归模型评价

Table 4 Evaluation of linear regression models for forest productivity and diversity in a typical mixed broadleaved-Korean pine forest

\begin{tabular}{lcccr}
\hline 模型参数 & $\begin{array}{c}\text { 物种多样性模型 } \\
\text { Model parameters }\end{array}$ & \multicolumn{3}{c}{ 功能多样性模型 } \\
\cline { 3 - 5 } & Species diversity motional diversity models & C \\
\hline$R^{2}$ & 0.169 & 0.269 & 0.188 & 0.377 \\
AIC & 2148.61 & 2047.73 & 2136.52 & 1932.43 \\
\hline
\end{tabular}

表中物种多样性模型包含了环境因子作为解释变量。功能多样性与群落加权平均值模型中包含 $\mathrm{A} 、 \mathrm{~B} 、 \mathrm{C}$ 三个独立模型(包含了环境因子作为解释变量), $\mathrm{A}$ 模型表示功能多样性指数与群落地上生产力的多元回归模型, B模型表示群落加权平均值与群落地上生产力的多元回归模型, C模型表示功能多样性 指数及群落加权平均值与群落地上生产力的多元回归模型。 $R^{2}$ 表示决定系数; AIC表示赤池信息量准则。

Functional diversity and CWM model include three independent models. Model A represents multiple regression models of functional diversity index and coarse woody productivity, Model B represents multiple regression models of community weight mean and coarse woody productivity, Model C represents multiple regression models of function diversity index, community-weight mean and coarse woody productivity. $R^{2}$ represents coefficient of determination; AIC represents the Akaike information criterion. 
表5 与生产力构建的线性模型中各变量相对重要性篎选结果

Table 5 Relative importance of variables in linear model constructed with productivity

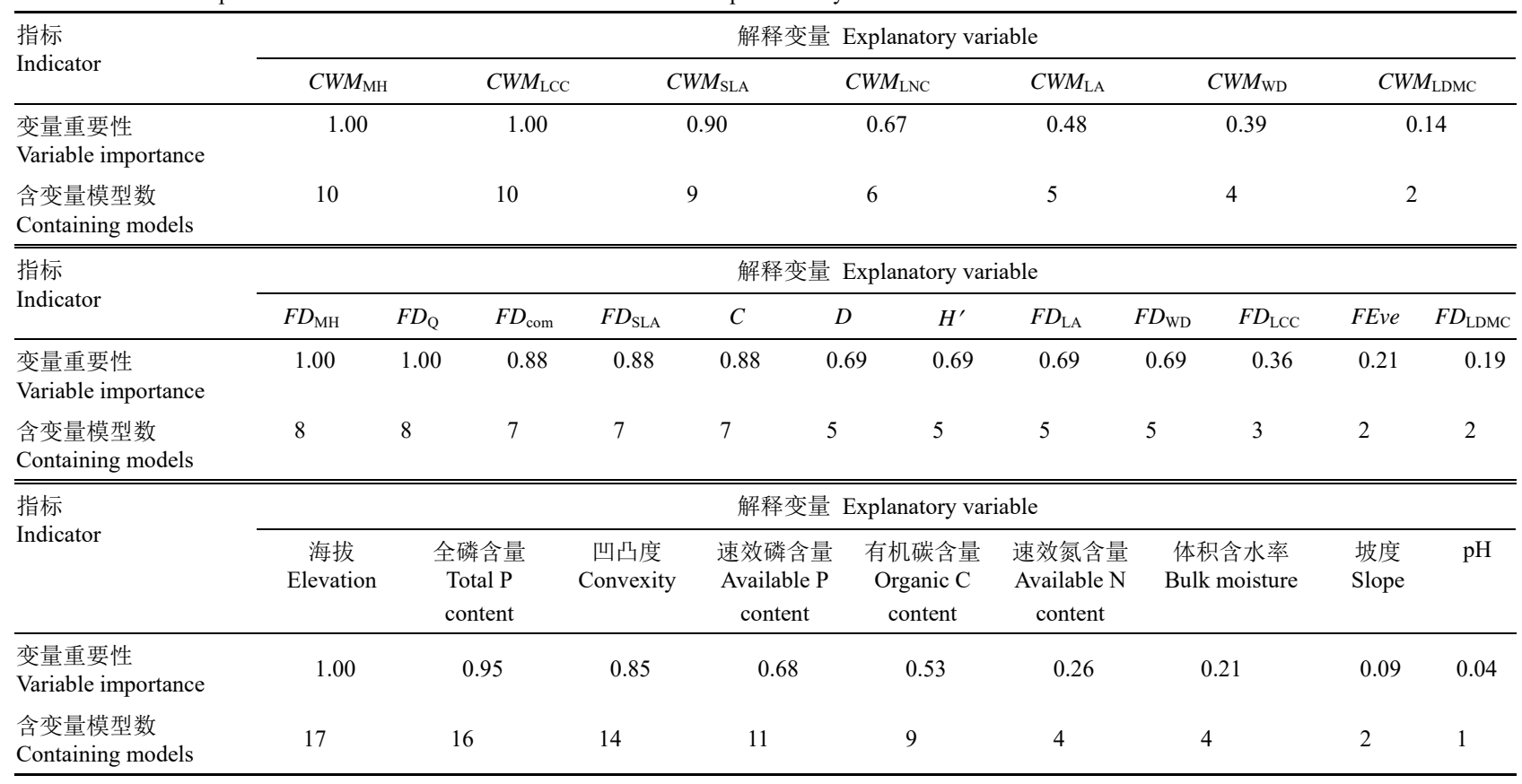

解释变量同表3。

Explanatory variables see Table 3 .

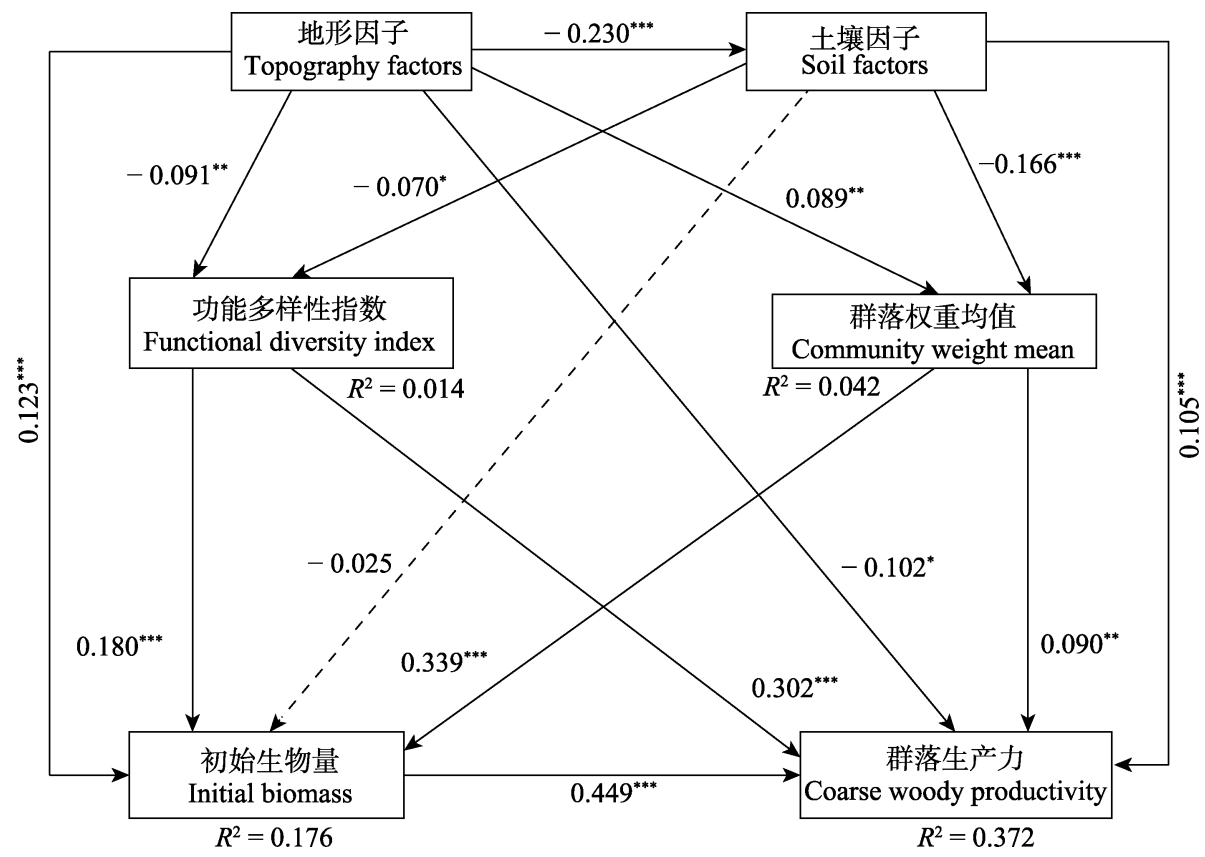

图5 结构方程模型分析环境因子、功能多样性、群落加权平均值和森林初始生物量对典型阔叶红松林群落生产力影响的结 果。图中实线表示作用路径显著, 虚线表示作用路径不显著。箭头旁边的值是具有相应统计意义的标准化路径系数。 $R^{2}$ 表示 由组合自变量解释的因变量的总变化。***, $p<0.001 ; * *, p<0.01 ; *, p<0.05$ 。

Fig. 5 Structural equation model (SEM) analysis of the effects of environmental factors, functional diversity, community-weight mean of functional traits and forest initial biomass on community woody productivity in a typical mixed broadleaved-Korean pine forest. Solid lines indicate significant paths, while dashed lines indicate insignificant paths. $R^{2}$ indicates the total variation in a dependent variable that is explained by the combined independent variables. ${ }^{* * *}, p<0.001$; **, $p<0.01$; $^{*}, p<0.05$.

具有显著的直接影响 $(p=0.002$, 路径系数为 0.090$)$, 且通过地上初始生物量具有极显著的间接影响 $(p<$ 0.001 , 路径系数为 0.152$)$, 总效应(直接和间接效应)
极显著 $(p<0.001)$, 路径系数为 0.242 (表 6$)$ 。同时, 生 产力还受地形因子和土壤因子的直接影响，作用大 小分别为 -0.102 和 0.105 ; 生产力受地上初始生物量

www.plant-ecology.com 
表6 基于结构方程模型森林地上生产力的直接、间接和总标准化效应

Table 6 Direct, indirect, and total standardized effects on the forest productivity, based on the structural equation models (SEM)

\begin{tabular}{|c|c|c|c|}
\hline \multirow{2}{*}{$\begin{array}{l}\text { 解释变量 } \\
\text { Explanatory variable }\end{array}$} & \multirow{2}{*}{$\begin{array}{l}\text { 对生产力的作用途径 } \\
\text { Pathway to productivity }\end{array}$} & \multicolumn{2}{|c|}{ 结构方程模型 SEM } \\
\hline & & 影响效应 Effect & $p$ \\
\hline \multirow[t]{6}{*}{ 地形因子 Topography factors } & 直接效应 Direct effect & -0.102 & 0.003 \\
\hline & 功能多样性的间接效应 Indirect effect via functional diversity & -0.027 & 0.009 \\
\hline & 群落加权平均值的间接效应 Indirect effect via CWMs & 0.008 & 0.043 \\
\hline & 初始生物量的间接效应 Indirect effect via initial biomass & 0.055 & $<0.001$ \\
\hline & 土壤因子的间接效应 Indirect effect via soil factors & -0.024 & 0.002 \\
\hline & 总效应 Total effect & -0.090 & $<0.001$ \\
\hline \multirow[t]{4}{*}{ 土壤因子 Edaphic factors } & 直接效应 Direct effect & 0.105 & $<0.001$ \\
\hline & 功能多样性的间接效应 Indirect effect via functional diversity & -0.021 & 0.045 \\
\hline & 群落加权平均值的间接效应 Indirect effect via CWMs & -0.015 & 0.008 \\
\hline & 总效应 Total effect & 0.069 & 0.019 \\
\hline \multirow[t]{3}{*}{ 功能多样性 Functional diversity } & 直接效应 Direct effect & 0.302 & $<0.001$ \\
\hline & 初始生物量的间接效应 Indirect effect via initial biomass & 0.081 & $<0.001$ \\
\hline & 总效应 Total effect & 0.383 & $<0.001$ \\
\hline \multirow{3}{*}{$\begin{array}{l}\text { 群落加权平均值 } \\
\text { Community-weighted mean functional traits }\end{array}$} & 直接效应 Direct effect & 0.090 & 0.002 \\
\hline & 初始生物量的间接效应 Indirect effect via initial biomass & 0.152 & $<0.001$ \\
\hline & 总效应 Total effect & 0.242 & $<0.001$ \\
\hline \multirow[t]{2}{*}{ 地上初始生物量 Initial standing biomass } & 直接效应 Direct effect & 0.449 & $<0.001$ \\
\hline & 总效应 Total effect & 0.449 & $<0.001$ \\
\hline
\end{tabular}

的极显著影响 $(p<0.001)$, 路径系数为 0.449 (图5; 表6)。

\section{3 讨论}

\section{1 功能多样性比物种多样性与生产力的关系更} 密切

越来越多的研究表明, 生态系统过程是由物种 的功能特性、物种的丰富度和物种的分布共同决定 的(McGill et al., 2006; Mouchet et al., 2010), 同时森 林生产力随多样性的增加而增加(Ruiz-Benito et al., 2014)。本研究得出相似的结果: 群落中物种丰富度 越高、功能性状差异越大, 其生产力越高(图1)。我 们的结果同样表明, 相较于物种多样性, 功能多样 性与生产力的关系更加密切(表4), 功能多样性比物 种多样性更好地解释了该群落生产力的变异。这可 能是因为物种数量和物种多样性所包含的关于物种 的特征信息少(Petchey et al., 2004), 或者忽略了物 种间功能性状的相似性和差异性(吕亭亭等, 2014)。

Petchey等(2004)首次证明功能多样性比物种多 样性能更有效地预测生物量的变化, Gazol 和 Camarero (2016) 指出功能多样性与生态系统生产力 的关系非常密切, 功能多样性是解释生态系统功能
变化的更有效的指标。相对于物种多样性, 功能多 样性有两个优点: 首先, 各物种具有相异的功能特 征，物种多样性无法解释物种间功能特性的差异, 而功能多样性能够综合不同物种的属性(Micheli \& Halpern, 2010); 其次, 功能性状可以表征生物从环 境中利用资源的能力(McGill et al., 2006)。功能性状 的多样性比物种多样性更好地反映不同物种的生态 位，因此能够更有效地揭示生产力的变异规律 (Petchey et al., 2004)。

\section{2 生态位互补效应比生物量比率效应更适于解 释阔叶红松林生产力的变异}

众多研究表明, 有关多样性对木本植物生物量 及其生产力的作用机理，既有生物量比率效应也有 生态位互补效应(Ruiz-Benito et al., 2014; Mensah et al., 2016), 但两种机制在生态系统过程中的作用不 相等(Mokany et al., 2008)。本研究中Rao二次熵指 数、功能分散指数在解释该群落生产力变异的能力 上明显优于群落加权平均值(图2; 表4); 在解释多 样性-生产力关系的最优结构方程模型中，两者均 与生产力显著相关, 功能多样性指数的总效应 (0.383)高于群落加权平均值的总效应(0.242)(表6)。 说明生态位互补效应和生物量比率效应共存，前者 
更适于解释阔叶红松林生产力的变异。

群落加权平均值用于评估优势性状对生产力的 影响, 而功能多样性指数则是量化该性状的变异性 对生产力的影响(Tobner et al., 2016)。在草本植物生 态系统中, 群落加权平均值能更好地解释生产力的 变异(Mokany et al., 2008), 而在欧洲地中海森林 (Ratcliffe et al., 2016)和广西马尾松(Pinus massoniana)林(黄小荣, 2018)则发现功能多样性指数更好 地解释了生产力的变异, 本研究结果与之一致。这 种不同群落类型的多样性-生产力关系结果的不一 致, 可能是方法上的差异, 包括选择不同的样地监 测周期和多样性指数(Dănescu et al., 2016); 也可能 是由不同生态系统类型和演替阶段造成的。当森林 的木本植物存在较大的功能性状差异时, 各物种具 有不同获取和利用资源的策略, 减少了生态位重叠, 生态位互补效应会减弱物种间对资源的竞争, 最大 化地利用光等有限资源, 从而提高生态系统生产力 (Díaz et al., 2007)。而在有生境过滤和扩散限制的群 落中, 优势物种的影响可能更大, 生态系统过程主 要取决于群落中优势物种的功能性状, 此时生物量 比率效应更为重要(Finegan et al., 2015)。

\section{3 生物多样性与生产力的关系受到生物和非生 物因素的共同作用}

生物多样性与生产力之间的关系直接和间接地 受多个因素的作用(Lohbeck et al., 2015)。本研究运 用结构方程模型评价了功能多样性、地上初始生物 量、土壤养分和地形条件对生产力的相对贡献。结 果显示, 地上初始生物量对生产力作用的总效应为 0.449 (表6), 在各因子中与生产力的相关路径系数 最大, 表明地上初始生物量是森林生产力最重要的 内在驱动因素, 即植被数量(即林分生物量)而非植 被质量(即功能多样性和功能性状组成) 是决定森林 生产力的主要因素。地形因子对土壤因子的作用效 应为- 0.230 , 并通过多样性、初始生物量对生产力 产生间接影响, 其对生产力的总效应为 -0.090 , 这 可能是由于海拔较高生境土壤水分和养分条件较差, 导致森林生产力下降。

在相对资源受限的环境中, 各物种采取不同的 资源吸收策略才能共存, 此时互补效应为生产力的 主要驱动力。在生物量较高的地区, 丰富的资源导 致互补效应的相对重要性降低, 多样性对生产力的 影响也将减弱(Lohbeck et al., 2015)。当群落中出现
非生物过滤(如环境过滤)时，只有适应相应环境条 件的物种才能够生存，导致物种间的性状差异减少， 形成性状聚集(Schellberg \& Pontes, 2012), 致使局 域范围内群落的优势物种表现出强烈的趋同现象。 因此, 生物多样性并非生态系统生产力的唯一驱动 因素，而是受非生物因素和其他生物因素的共同影 响(Lohbeck et al., 2015)。

在小兴安岭阔叶红松林中, 物种多样性、功能 多样性对森林生产力均有一定的促进作用; 功能多 样性能够更好地解释群落生产力的变异。功能多样 性对生产力的效应主要通过Rao二次熵指数、基于 最大树高的功能分散指数产生; 群落加权平均值效 应主要由叶片碳含量、比叶面积的群落加权平均值 产生; 功能多样性的效应大于群落加权平均值效 应。生物因素与非生物因素共同调控多样性-生产力 关系。本研究的结果为更好地了解温带森林生态系 统中生物多样性与生产力之间的复杂关系提供了科 学依据, 对有效增加生产力具有重要意义。

\section{参考文献}

Ali A, Yan ER, Chang SX, Cheng JY, Liu XY (2017). Community-weighted mean of leaf traits and divergence of wood traits predict aboveground biomass in secondary subtropical forests. Science of the Total Environment, 574, 654-662.

Bartoń K (2018). Package 'MuMIn'. https://cran.r-project.org/web/ packages/MuMIn/MuMIn.pdf. Cited: 2018-07-21.

Cardinale BJ, Duffy JE, Gonzalez A, Hooper DU, Perrings C, Venail P (2012). Biodiversity loss and its impact on humanity. Nature, 489, 59-67.

Cardinale BJ, Gonzalez A (2011). The functional role of producer diversity in ecosystems. American Journal of Botany, 98, 572-592.

Chang LW, Zeleny D, Li CF, Chiu ST, Hsieh CF (2013). Better environmental data may reverse conclusions about nicheand dispersal-based processes in community assembly. Ecology, 94, 2145-2151.

Chave J, Condit R, Lao S, Caspersen JP, Foster RB, Hubbell SP (2003). Spatial and temporal variation of biomass in a tropical forest: Results from a large census plot in Panama. Journal of Ecology, 91, 240-252.

Chen CG, Zhu JF (1989). A Handbook for Main Tree Species Biomass in Northeast China. China Forestry Publishing House, Beijing. [陈传国, 朱俊风 (1989). 东北主要林木 生物量手册. 中国林业出版社, 北京.]

Chen DM, Cheng JH, Chu PF, Mi J, Hu SJ, Xie YC, Tuvshintogtokh I, Bai YF (2016). Effect of diversity on biomass

www.plant-ecology.com 
across grasslands on the Mongolian Plateau: Contrasting effects between plants and soil nematodes. Journal of Biogeography, 43, 955-966.

Cornelissen JHC, Lavorel S, Garnier E, Diaz S, Buchmann N, Gurvich DE, Reich PB, ter Steege H, Morgan HD, van der Heijden MGA, Pausas JG, Poorter H (2003). A handbook of protocols for standardised and easy measurement of plant functional traits worldwide. Australian Journal of Botany, 51, 335-380.

Díaz S, Lavorel S, Bello FD, Quétier F, Grigulis K, Robson TM (2007). Incorporating plant functional diversity effects in ecosystem service assessments. Proceedings of the National Academy of Sciences of the United States of America, 104, 20684-20689.

Dănescu A, Albrecht AT, Bauhus J (2016). Structural diversity promotes productivity of mixed, uneven-aged forests in southwestern Germany. Oecologia, 182, 319-333.

Edwards JR, Lambert LS (2007). Methods for integrating moderation and mediation: A general analytical framework using moderated path analysis. Psychological Methods, 12, $1-22$.

Finegan B, Peña-Claros M, de Oliveira A, Ascarrunz N, Bret-Harte MS, Carreño-Rocabado G, Casanoves F, Díaz S, Eguiguren Velepucha P, Fernandez F, Licona JC, Lorenzo L, Salgado Negret B, Vaz M, Poorter L, Canham C (2015). Does functional trait diversity predict above-ground biomass and productivity of tropical forests? Testing three alternative hypotheses. Journal of Ecology, 103, 191-201.

Fox J (2008). Applied Regression Analysis and Generalized Linear Models. 2nd edn. Sage Publications, Thousand Oaks, USA.

Fox J, Monette G (1992). Generalized collinearity diagnostics. Journal of the American Statistical Association, 87, 178-183.

Gazol A, Camarero JJ (2016). Functional diversity enhances silver fir growth resilience to an extreme drought. Journal of Ecology, 104, 1063-1075.

Grace JB, Anderson TM, Seabloom EW, Borer ET, Adler PB, Harpole WS, Hautier Y, Hillebrand H, Lind EM, Partel M, Bakker JD, Buckley YM, Crawley MJ, Damschen EI, Davies KF, Fay PA, Firn J, Gruner DS, Hector A, Knops JM, MacDougall AS, Melbourne BA, Morgan JW, Orrock JL, Prober SM, Smith MD (2016). Integrative modelling reveals mechanisms linking productivity and plant species richness. Nature, 529, 390-393.

Grime JP (1998). Benefits of plant diversity to ecosystems: Immediate, filter and founder effects. Journal of Ecology, 86, 902-910.

Howard AL (2013). Handbook of structural equation modeling. Structural Equation Modeling-a Multidisciplinary Journal, 20, 354-360.

Huang XR (2018). Relationship between plant functional diver- sity and productivity of Pinus massoniana plantations in Guangxi. Biodiversity Science, 26, 690-700. [黄小荣 (2018). 广西马尾松林植物功能多样性与生产力的关系. 生物多样性, 26, 690-700.]

Laliberté E, Legendre P (2010). A distance-based framework for measuring functional diversity from multiple traits. Ecology, 91, 299-305.

Lin DM, Lai JS, Muller-Landau HC, Mi XC, Ma KP (2012). Topographic variation in aboveground biomass in a subtropical evergreen broad-leaved forest in China. PLOS ONE, DOI: 10.1371/journal.pone.0048244.

Lohbeck M, Poorter L, Martinez-Ramos M, Bongers F (2015). Biomass is the main driver of changes in ecosystem process rates during tropical forest succession. Ecology, 96, 1242-1252.

Lü TT, Wang P, Yan H, Zhang W, Liao GX, Jiang HB, Zou CL, Sheng LX (2014). Relationship between functional diversity and productivity in meadow and marsh plant communities. Chinese Journal of Plant Ecology, 38, 405-416. [吕亭亭, 王平, 燕红, 张稳, 廖桂项, 姜海波, 邹畅林, 盛连喜 (2014). 草甸和沼泽植物群落功能多样 性与生产力的关系. 植物生态学报, 38, 405-416.]

McGill BJ, Enquist BJ, Weiher E, Westoby M (2006). Rebuilding community ecology from functional traits. Trends in Ecology and Evolution, 21, 178-185.

Mensah S, Veldtman R, Assogbadjo AE, Glèlè KR, Seifert T (2016). Tree species diversity promotes aboveground carbon storage through functional diversity and functional dominance. Ecology and Evolution, 6, 7546-7557.

Micheli F, Halpern BS (2010). Low functional redundancy in coastal marine assemblages. Ecology Letters, 8, 391-400.

Mokany K, Ash J, Roxburgh S (2008). Functional identity is more important than diversity in influencing ecosystem processes in a temperate native grassland. Journal of Ecology, 96, 884-893.

Mouchet MA, Villeger S, Mason NWH, Mouillot D (2010). Functional diversity measures: An overview of their redundancy and their ability to discriminate community assembly rules. Functional Ecology, 24, 867-876.

Oksanen J, Kindt R, Legendre P, O’Hara B, Simpson GL, Slymos P, Stevens MHH, Wagner H (2009). The vegan Package. http://cran.r-project.org/web/packages/vegan/vegan.pdf. Cited: 2019-02-04.

Ouyang S, Xiang W, Wang X, Zeng Y, Lei P, Deng X, Peng C (2016). Significant effects of biodiversity on forest biomass during the succession of subtropical forest in south China. Forest Ecology and Management, 372, 291-302.

Petchey OL, Hector A, Gaston KJ (2004). How do different measures of functional diversity perform? Ecology, 85, 847-857.

Ratcliffe S, Liebergesell M, Ruiz-Benito P, Madrigal González J, Muñoz Castañeda JM, Kändler G, Lehtonen A, 
Dahlgren J, Kattge J, Peñuelas J, Zavala MA, Wirth C (2016). Modes of functional biodiversity control on tree productivity across the European continent. Global Ecology and Biogeography, 25, 251-262.

Rosseel Y (2012). lavaan: An R package for structural equation modeling. Journal of Statistical Software, 48, 1-36.

Ruiz-Benito P, Gomez-Aparicio L, Paquette A, Messier C, Kattge J, Zavala MA (2014). Diversity increases carbon storage and tree productivity in Spanish forests. Global Ecology and Biogeography, 23, 311-322.

Schellberg J, Pontes LDS (2012). Plant functional traits and nutrient gradients on grassland. Grass and Forage Science, 67, 305-319.

Sun RY, Li QF, Niu CJ, Lou AR (2002). Basic Ecology. Higher Education Press, Beijing. [孙儒泳, 李庆芬, 牛翠娟, 娄 安如 (2002). 基础生态学. 高等教育出版社, 北京.]

Tilman D (1997). Distinguishing between the effects of species diversity and species composition. Oikos, 80, 185.

Tobner CM, Paquette A, Gravel D, Reich PB, Williams LJ,
Messier C (2016). Functional identity is the main driver of diversity effects in young tree communities. Ecology Letters, 19, 638-647.

Van MT, Peña-Claros M, Ascarrunz N, Arets EJMM, Licona JC, Toledo M, Poorter L (2017). Abiotic and biotic drivers of biomass change in a neotropical forest. Journal of Ecology, 105, 1223-1234.

Wu Z, Raven P (1994-2009). Flora of China. Science Press and Missouri Botanical Garden Press, Beijing and St Louis.

Zhang Y, Chen HYH (2015). Individual size inequality links forest diversity and above-ground biomass. Journal of Ecology, 103, 1245-1252.

Zhang Y, Chen HYH, Reich PB (2012). Forest productivity increases with evenness, species richness and trait variation: A global meta-analysis. Journal of Ecology, 100, 742-749.

责任编委: 李意德 责任编辑: 李 敏 